\title{
Liver imaging reporting and data system category on magnetic resonance imaging predicts recurrence of hepatocellular carcinoma after liver transplantation within the Milan criteria: a multicenter study
}

\author{
Sunyoung Lee ${ }^{1}$, Kyoung Won Kim² \\ 1Department of Radiology, Severance Hospital, Seoul, Korea
${ }^{2}$ Department of Radiology, University of Ulsan College of Medicine, Seoul, Korea
}

Background: This study aimed to investigate the association between Liver Imaging Reporting and Data System (LI-RADS) category and recurrence of hepatocellular carcinoma (HCC) after primary liver transplantation (LT) within the Milan criteria.

Methods: This multicenter retrospective study included 140 recipients who underwent living donor LT (LDLT) for treatment-naïve HCC and pretransplant contrast-enhanced magnetic resonance imaging (MRI) between 2009 and 2013. LI-RADS categories were assigned using LI-RADS version 2018. Recurrence-free survival (RFS) and associated factors were evaluated using Cox proportional hazards regression analysis, Kaplan-Meier analysis, and log-rank test. Histological grading and microvascular invasion $(\mathrm{MVI})$ were analyzed on the pathologic examinations of explanted livers.

Results: The overall 1-, 3-, 5-, and 7-year RFS rates were $95.6 \%, 92.6 \%, 90.2 \%$, and $89.3 \%$, respectively. In the multivariable analysis, independent predictors of recurrence included HCCs categorized as LR-M (hazard ratio [HR], 18.68; 95\% confidence interval [CI], 5.79-60.23; $\mathrm{P}<0.001)$ and the largest tumor size of $\geq 3 \mathrm{~cm}$ on MRI (HR, 4.18; 95\% Cl, 1.42-12.37; $\mathrm{P}=0.010)$. The 5-year RFS rate was significantly lower in patients with HCCs categorized as LR-M than in those with HCCs categorized as LR-5 or 4 (LR-5/4) (36.9\% vs. $95.8 \%$, respectively; $P<0.001)$. HCCs categorized as LR-M exhibited significantly more MVI than HCCs categorized as LR-5/4 (57.1\% vs. $17.5 \%$, respectively; $P=0.002)$.

Conclusions: Patients with HCCs categorized as LR-M using LI-RADS version 2018 may have a worse prognosis after primary LT within the Milan criteria than those with HCCs categorized as LR-5/4. 